\title{
Artificial Intelligence tools in radiological practice
}

Julián Dorado de la Calle

An introduction to the different types of Artificial Intelligence (AI) systems is made and the best characteristics of each type are discussed for use in the medical environment. A brief historical introduction to the development of Al systems in medicine is made commenting on the successes and failures that occurred.

Next, the most active fields of application in medicine of Al are studied in depth.

We first speak of prioritization systems for radiologists' work lists, calculating scores for each patient so that the most serious patients are reported as a priority.

It is discussed later on Image Enhancement systems in two senses. Those systems that are placed between the medical modalities and the storage systems (PACS) to increase the quality of the images by reducing the radiation doses received by the patient. Also on the systems that highlight and indicate pathologies in the images for the radiologist to review and validate the indication, also learning from the doctor's feedback.

Finally, there is talk about automatic medical reporting systems that examine images to identify pathologies and are capable of writing reports and selecting key images. These systems also work by reinforcement using Feedback from the radiologist. 